\title{
Curcumin and Zinc Co-Supplementation Along With a Loss-Weight Diet Can Improve Lipid Profiles in Subjects with Pre-Diabetes: A Multi-Arm, Parallel- Group, Randomized, Double-Blind Placebo- Controlled Phase 2 Clinical Trial
}

\section{Majid Karandish}

Ahvaz Jundishapur University: Ahvaz Jondishapour University of Medical Sciences

\section{Hassan Mozaffari-khosravi}

Yazd University of Medical Science: Shahid Sadoughi University of Medical Sciences and Health

Services

Seyed Mohammad Mohammadi

Yazd University of Medical Science: Shahid Sadoughi University of Medical Sciences and Health

Services

\section{Bahman Cheraghian}

Ahvaz Jundishapur University: Ahvaz Jondishapour University of Medical Sciences

Maryam Azhdari ( $\square$ azhdari_mar@yahoo.com )

Shahid Sadoughi University of Medical Sciences and Health Services https://orcid.org/0000-00032110-9817

\section{Research}

Keywords: Curcumin, Zinc, Dietary intake, Liver enzymes, Lipid profiles, Pre-diabetes.

Posted Date: October 1st, 2021

DOl: https://doi.org/10.21203/rs.3.rs-948451/v1

License: (9) This work is licensed under a Creative Commons Attribution 4.0 International License. Read Full License 


\section{Abstract}

Background: Diabetes is one of the major public health concerns. Pre-diabetes can increase the risk of developing some non-communicable diseases such as type 2 diabetes. Given the increasing trend of prediabetes, it is critical to control it and prevent its complications. Curcumin is a major bioactive component of turmeric. Zinc is an antioxidant nutrient. The present trial aimed to evaluate the effect of curcumin and zinc co-supplementation along with a loss-weight diet on serum lipid profiles in overweight or obese patients with pre-diabetes.

Methods: Eighty-four participants were randomized to four groups (curcumin (500 mg/day), zinc (30 $\mathrm{mg} /$ day), "curcumin \& zinc", and placebo) for 90 days. Serum total cholesterol (TC), low-density lipoprotein (LDL), high-density lipoprotein (HDL), triglycerides (TG), non-HDL, HDL/LDL ratio, weight, BMI, waist circumstance (WC), hip circumstance $(\mathrm{HC})$, physical activity (PA) and dietary intake were determined pre and post-intervention. This study will be conducted at Yazd Diabetes Research Clinic, Shahid Sadoughi University of Medical Sciences.

Results: Totally, 82 participants were included in the final analysis. After the adjusted PA effect, changes in serum TG (adjusted $p=0.001$ ), LDL (adjusted $p=0.035$ ), non-HDL (adjusted $p=0.003$ ), HDL/LDL ratio (adjusted $p=0.002$ ), and HDL (adjusted $p<0.0001$ ) revealed a significant difference between the groups. However, the changes in weight (adjusted $p=0.004$ ) and BMI (adjusted $p=0.006$ ) were significant but the changes in dietary intake, PA, WC, and $\mathrm{HC}$ were non-significant (adjusted $\mathrm{p} \geq 0.05$ ). Despite that there was a significant difference for post-intervention HDL levels (adjusted $p=0.016$ ), other lipid profiles showed no significant difference (adjusted $p \geq 0.05$ ).

Conclusion: The beneficial effects of "curcumin \& zinc" co-supplementation was reported for the changes of some lipid profiles (TG, LDL, HDL, non-HDL, and HDL to LDL ratio), BMI, and weight with no positive effects on TC, dietary intake, PA, WC, and HC. Therefore, it may play a potential role in the prevention of macro and microvascular complications.

Trial registration: The project is a registered clinical trial (Registration number: IRCT20190902044671N1, Iranian Registry of Clinical Trials (IRCT), registered October 11, 2019.

\section{Introduction}

Prediabetes status is defined as impaired glucose tolerance (IGT), impaired fasting glucose (IFG), and/or $\mathrm{HbA}_{1} \mathrm{C}$ 5.7-6.4\% ${ }^{1}$. Some complications of prediabetes include macro vascular (cardiovascular disease (CVD), stroke, peripheral artery disease), microvascular (retinopathy, neuropathy, and nephropathy), and type 2 diabetes mellitus (T2DM) ${ }^{1,2}$. The prevalence of prediabetes among some populations was related to abdominal or visceral obesity, dyslipidemia with high triglycerides (TG), high low-density lipoprotein cholesterol (LDL-C), high total cholesterol (TC), low high-density lipoprotein-cholesterol (HDL-C), and/or hypertension ${ }^{1,3,4}$. Moreover, the high prevalence of obesity, dyslipidemia, and T2DM along with 
cardiovascular complications was reported in the past decade. The physiological, metabolic, or/ and biochemical characteristics are abnormal in pre-diabetic patients. Since the adverse changes in both lipid and glucose concentrations were reported in prediabetes and T2DM, the target of the novel therapeutic approaches is the simultaneous improvement of glucose and lipid control. Therefore, early diagnosis and effective intervention are critical to control prediabetes and delay its complications ${ }^{5}$. Physical activity (PA) and dietary modifications can consider as the key lifestyle interventions. The pharmacological interventions usually apply in pre-diabetic patients without a positive response to lifestyle interventions ${ }^{2}$. Recent researches have shown too much attention to phytochemicals and the antioxidant trace elements to treat and control some diseases (T2DM, pre-diabetes, metabolic syndrome, CVD, and chronic venous insufficiency $(\mathrm{CVI}))^{6-11}$.

Curcumin (diferuloylmethane) is a bioactive constituent of Curcuma longa L. (Zingiberaceae), commonly known as turmeric with diverse pharmacological and biological properties ${ }^{7}$. Curcumin has shown beneficial effects on glycemic parameters (fasting plasma glucose (FPG), 2-hour postprandial (2hpp), $\mathrm{HbA}_{1} \mathrm{C}$, insulin, insulin sensitivity (IS) and insulin resistance (IR)) in pre-diabetic patients ${ }^{11}$, lipid profiles (TG, TC, LDL-C, and HDL-C) and blood pressure (BP) in T2DM ${ }^{8}$ and metabolic syndrome ${ }^{6}$. The finding of a meta-analysis of randomized controlled trials showed curcumin may play a protective role in patients at risk of CVD by improving serum lipid levels ${ }^{10}$.

Zinc is a trace element that has health benefits in the various aspects of metabolism through antioxidant and anti-inflammatory properties, and cofactor of many enzymes ${ }^{12-14}$. Zinc apart from its role in improving glycemic markers in patients with pre-diabetes ${ }^{11}$ and T2DM ${ }^{15}$, play a notable role in lipid metabolism ${ }^{16}$ that can control CVD, one of the current complications in pre-diabetes and diabetes. However, the findings of some trials or meta-analyses of trials indicated that zinc supplementation improves some lipid profiles, some studies did not show it ${ }^{17-19}$.

On the one hand, the results of the effect of curcumin or zinc were inconsistent in previous studies, and on the other hand, there was no trial with aim of evaluating the effect of curcumin and zinc cosupplementation on lipid profiles in pre-diabetic patients. The present multi-arm, parallel-group, randomized, double-blind placebo-controlled phase 2 clinical trial aimed to evaluate the effect of curcumin and zinc co-supplementation along with loss-weight diet on serum lipid profiles (TG, LDL, HDL, TC, non-high-density lipoprotein cholesterol (non-HDL), and HDL to LDL ratio) in overweight or obese patients with pre-diabetes.

\section{Methods}

The details of the method and materials were previously published 20 .

\section{Participants and sampling}


All 84 eligible pre-diabetic adults with written informed consent recruited from Yazd Diabetes Research Clinic and enrolled in the present randomized, clinical trial (RCT) for 90 days. The inclusion criteria were included women or men (18-50 years old for men and 18 years- before menopause for women) with prediabetes according to the ADA guidelines ${ }^{1}$ and $25<\mathrm{BMI}>35$. Patients were excluded from the trial if a diagnosis of any types of malignancies/cancers, cardiovascular, kidney, lung, endocrine, autoimmune, inflammatory, and neurological diseases, and/ or hypertension, taking BP, glucose or lipid-lowering drugs; taking multivitamin-mineral supplements for three months before or during the intervention; a history of weight loss surgery in the last year, a weight-loss plan in the last 3 months; receiving from a weight-loss medicine or program; lactating, pregnant or planning to get pregnant; unwillingness/ the compliance of less than $80 \%$ during the intervention, or no signed informed consent.

Considering that the present study was a part of a comprehensive research, the sample size was measured for all variables and the largest sample size was considered. The sample size was calculated using both WinPepi statistical program (Version 11.4: Abramson, 2011) and a parallel design randomized controlled trial formula (formula was previously published) ${ }^{20}$.Twenty-one participants in each group were required (80\% power at 0.05 significance level and accounting for a drop-out rate of $10 \%)^{20}$.

\section{Study setting and design}

This multi-arm, parallel-group, randomized, double-blind, placebo-controlled, phase 2 clinical trial was reported according to the CONSORT statement (Consolidated Standards for Reporting Trials). The method of block randomization with a block size of 4 using a computer-generated random number sequence and the allocation concealment (assigning the unique codes to the eligible participant) by a blinded independent statistician and the pharmacist (who was not involved during the study), respectively. All the assessments in the trial were made by the investigators blinded to the treatment allocation. The participants were not informed about the type of used supplement. The supplements were delivered to the participants according to the allocation on the 1st, 30th, and 60th day.

The eligible participants randomized into four parallel groups, 1) the curcumin group: curcumin supplement (500 mg-BCM95/ Curcugreen capsule, M/s Arjuna Natural Pvt Ltd., India) and placebo for zinc (lactose); 2) the zinc group: zinc supplement (30 mg zinc in form of zinc gluconate tablet, Dineh company, Iran) and the placebo for curcumin (roasted rice powder) 3) the "curcumin \& zinc" group: curcumin and zinc supplement; 4) the placebo group: the placebo for both curcumin and zinc. Dosages were selected based on the previous studies ${ }^{21,22}$. The placebo was identical in texture, weight, and appearance to its active supplement. A curcumin capsule (supplement or placebo) and a zinc tablet (supplement or placebo) were prescript once daily after and before breakfast for 90 days, respectively. All participants have followed a standard, individualized hypo-caloric diet designed with at least a $7 \%$ weight loss including $45-55 \%, 25-35 \%$, and $10-20 \%$ of their calories from carbohydrate, fat, and protein, respectively and PA for 150 minutes per week for improving lifestyle. The nutritional modifications, including adherence to the diet and avoiding excessive intake of high-fat and/or sugar products, were recommended by a nutritionist. 
This trial approved by the Medical Ethics Committee of Ahvaz Jundishapur University of Medical Sciences, Ahvaz, Iran; (Ethical code: IR.AJUMS.REC.1398.504) and it is a prospectively registered in Iranian Registry of Clinical Trials (Registration number: IRCT20190902044671N1.

\section{Follow-up}

In order to control the participants for taking supplements and placebo, they were followed-up using messaging tools or the telephone, daily or weekly. The lifestyle modifications were individually recommended for all participants who were visited at the end of 30-day periods. Moreover, pill counts were recorded every 30 days to assess adherence to the supplement. All adverse events were documented and reported to the Data Monitoring Committee and the Ethics Committee of the Ahvaz Jundishapur University of Medical Sciences.

\section{Data collection and outcome measures}

The general demographic and medical history data (age, gender, education levels, duration of prediabetes, and family history of pre-diabetes or hypertension) were obtained from each participant by interview. The measurement of weight and height were recorded to assess body mass index (BMI) $\left(\mathrm{kg} / \mathrm{m}^{2}\right)$ which calculated by this equation: weight $(\mathrm{kg}) /$ height $\left(\mathrm{m}^{2}\right)$. The waist and hip circumstances were assessed by a non-elastic and flexible tape.

The short form of the International Physical Activity Questionnaire (IPAQ-SF) was used for the evaluation of PA. PA was reported as metabolic equivalents (METs)-minutes per week (MET-min/week) and categorized into three groups including active (PA> $3000 \mathrm{MET}-\mathrm{min} /$ week), moderate (PA $=600-3000 \mathrm{MET}-$ $\mathrm{min} /$ week), and inactive (PA< $600 \mathrm{MET}-\mathrm{min} /$ week) ${ }^{23}$.

Diastolic Blood Pressure (DBP) and Systolic Blood Pressure (SBP) were measured the baseline of the study according to the American Heart Association protocol (blood pressure was assessed in a quiet place after 5 minutes of rest in sitting status without crossed legs and unsupported back and arms) ${ }^{24}$. The side effects of supplements were recorded during the study and also liver enzymes were evaluated to monitor possible side effects of supplements.

Blood samples were collected in an EDTA tube and centrifuged to obtain serum. Serum TG, HDL, TC, FPG $(\mathrm{mg} / \mathrm{dL})$ levels, $\mathrm{HbA1C}(\%)$, alanine transaminase (ALT), and aspartate transaminase (AST) were measured immediately after sampling. The values of non-HDL, HDL/LDL ratio, and LDL-C were calculated by the following the formula: TC minus HDL-C, HDL $(\mathrm{mg} / \mathrm{dL})$ divided by LDL $(\mathrm{mg} / \mathrm{dL})$, and Friedewald formula (TC $(\mathrm{mg} / \mathrm{dL})-\mathrm{HDL}-\mathrm{c}(\mathrm{mg} / \mathrm{dL})-\mathrm{TG}(\mathrm{mg} / \mathrm{dL}) / 5)$, respectively ${ }^{25}$.

The outcomes including serum lipid profiles and anthropometric measurements which were measured at the baseline and day 90 after the intervention.

\section{Statistical analysis}


All analyses were performed using a statistical software package (SPSS), version 22.0 (SPSS, Inc, Chicago, Illinois, USA). Statistical significance was determined at $p<0.05$. Normal distribution and the homogeneity of variances for quantitative variables were checked by Kolmogorov-Smirnov test and Leven's tests, respectively. Data were reported as mean \pm standard deviation (SD) and median (interquartile range) for normally and non-normally distributed data, respectively. Within-group comparisons were performed using a paired-samples t-test. Between-group comparisons for variables were carried out using a one-way ANOVA with post hoc (LSD) analysis test and Kruskal-Wallis for normally and non-normally distributed data, respectively. The changes (post-pre intervention) calculated based on the difference of variables from the baseline to the end of the study. However, there was any significant difference for pre-intervention, post-intervention, ${ }^{11}$ and changes of dietary intake (supplementary file, Table S.2), a significant difference was previously reported in PA levels at the baseline and changes in $\mathrm{BMI}$ and weight. Therefore, the adjustment was applied using univariate analysis of covariance (ANCOVA) and a general linear model to control the effects of the baseline PA or/and changes of BMI and weight from the baseline.

\section{Results}

A total of 82 participants completed the 90 days of the trial. Two patients lost the follow-up due to poor compliance and nausea and severe abdominal pain in the "curcumin \& zinc" and placebo group (1 patient in each group), respectively. Figure 1 showed the flow diagram of the study. The mild side effects including headache $(n=2)$ in curcumin group, headache $(n=1)$ in zinc group, headache $(n=1)$, nausea $(n=2)$, dizziness $(n=1)$ in "curcumin \& zinc" group, and headache $(n=1)$, nausea $(n=1)$, hearing impairment $(n=1)$ in placebo group were reported by the participants.

The mean duration of pre-diabetes (months) and age (year) of participants was reported $2.31 \pm 1.37$ and $36.04 \pm 6.61$, respectively. As set out in Table 1, the general and clinical characteristics of the participants were not found any significant difference between the four groups at the baseline except PA levels $(p=$ 0.03). Moreover, there was no significant difference in pre-intervention biochemical measurements of the participants between groups (supplementary file, Table S.1). 
Table 1

General and Clinical characteristics of the participants at the baseline.

Variables

Groups

$\begin{array}{llll}\text { Placebo Curcumin Zinc } & \text { Curcumin \& } \\ \text { Zinc }\end{array}$

Pvalue

$\mathrm{N}=\mathbf{2 0}$

$\mathrm{N}=21$

$\mathrm{N}=21$

$\mathrm{N}=20$

\section{Qualitative variables}

Age (year)

$34.19 \pm 7.03$

$36.95 \pm$

$38.19 \pm$

$34.48 \pm 6.45$

0.14

Weight $(\mathrm{kg})$

$8467 \pm 9.78$

$82.6 \pm 8.03$

$81.81 \pm$

13.08

$80.57 \pm$

10.17

Body mass index $\left(\mathrm{kg} / \mathrm{m}^{2}\right)$

$30.97 \pm 2.33$

$30.46 \pm$

$29.5 \pm 2.82$

$29.95 \pm 2.56$

$0.32 *$

2.75

Waist circumstance $(\mathrm{cm})$

$108.01 \pm$

$107.44 \pm$

$103.39 \pm$

11.75

$106.77 \pm$

10.19

$0.493 *$

10.18

9.9

$109.79 \pm$

$108.88 \pm$

112.47
6.84

6.04

5.15

Physical Activity

445 (396-

853.88)

$990(495-$

1860)

396 (396-

1440)

396 (396-

982)

$0.65^{\star}$

Systolic blood pressure

$(\mathrm{mmHg})$

$11.56 \pm 0.9$

$11.77 \pm$

0.69

$11.57 \pm$

$11.45 \pm 0.82$

0.437

Diastolic blood pressure $(\mathrm{mmHg})$

$7.43 \pm 0.43$

$7.5 \pm 0.36$

0.78

$0.242^{*}$

Fat (gr)

$30.47 \pm 3.85$

$32.03 \pm$

3.42

$7.3 \pm 0.51$

$7.33 \pm 0.42$

0.644

$0.03^{\#}$

Quantitative variables

Gender (Female)

$12(60)$

$16(76.2)$

15 (71.5)

$13(65)$

$0.837^{\#}$

\section{Education levels}

University education

$10(50)$

$14(66.7)$

$12(57.1)$

$12(60)$

$0.585^{\#}$

Data are presented as mean \pm standard deviation (SD) for quantitative variables and frequency (\%) for qualitative variables.

*P-value was calculated for the comparison variables between four groups using one-way analysis of variance (one-way ANOVA).

\# P-value was calculated for the comparison variables between four groups using Kruskal-Wallis.

P-value $<0.05$ was considered significant. 


\begin{tabular}{|c|c|c|c|c|c|}
\hline \multirow[t]{4}{*}{ Variables } & \multicolumn{4}{|l|}{ Groups } & \multirow{4}{*}{$\begin{array}{l}\mathrm{P} \text { - } \\
\text { value }\end{array}$} \\
\hline & \multirow{3}{*}{$\begin{array}{l}\text { Placebo } \\
\mathrm{N}=20\end{array}$} & \multirow{3}{*}{$\begin{array}{l}\text { Curcumin } \\
\mathrm{N}=21\end{array}$} & \multirow{3}{*}{$\begin{array}{l}\text { Zinc } \\
\mathrm{N}=21\end{array}$} & Curcumin \& & \\
\hline & & & & & \\
\hline & & & & $\mathrm{N}=\mathbf{2 0}$ & \\
\hline Diploma & $6(30)$ & $6(28.6)$ & $4(19.1)$ & $6(30)$ & \\
\hline Under diploma & $4(20)$ & $1(4.8)$ & $5(23.8)$ & $2(10)$ & \\
\hline \multicolumn{6}{|c|}{$\begin{array}{l}\text { Data are presented as mean } \pm \text { standard deviation (SD) for quantitative variables and frequency (\%) } \\
\text { for qualitative variables. }\end{array}$} \\
\hline \multicolumn{6}{|c|}{$\begin{array}{l}\text { *P-value was calculated for the comparison variables between four groups using one-way analysis of } \\
\text { variance (one-way ANOVA). }\end{array}$} \\
\hline \multicolumn{6}{|c|}{ \# P-value was calculated for the comparison variables between four groups using Kruskal-Wallis. } \\
\hline P-value $<0.0$ & if & & & & \\
\hline
\end{tabular}

For data with both adjusted and crude analyses, only adjusted analyses were reported. Please refer to tables for crude analysis. No significant difference was shown for changes (pre-post) in dietary intake (energy, protein, carbohydrate, and fat) (adjusted $p \leq 0.05$ ) (supplementary file, Table S.2).

The comparison of changes in liver enzymes, anthropometry measurements, and physical activity was depicted in Table 2. The changes in AST, ALT, WC, HC, and PA were non-significant after adjusting the baseline PA effect (adjusted $p \geq 0.05$ ). After removing PA effects, the changes of weight and BMI were significant (adjusted $p=0.004$ and 0.006 , respectively). In comparison to the placebo, all three groups receiving the supplement showed significant differences for the changes of weight and BMI after adjusting PA effects (supplementary files, Table S.3). 
Table 2

Comparison of changes in liver enzymes, anthropometry measurements, and physical activity between the groups.

\begin{tabular}{|c|c|c|c|c|c|c|}
\hline \multirow[t]{3}{*}{ Variables } & \multicolumn{4}{|l|}{ Groups } & \multirow[t]{3}{*}{ p } & \multirow{3}{*}{$\begin{array}{l}\text { Adjusted } \\
\mathrm{P}^{*}\end{array}$} \\
\hline & Placebo & \multirow{2}{*}{$\mathrm{N}=21$} & \multirow{2}{*}{$\begin{array}{l}\text { Zinc } \\
N=21\end{array}$} & \multirow{2}{*}{$\begin{array}{l}\text { Zinc \& } \\
\text { curcumin } \\
\mathrm{N}=20\end{array}$} & & \\
\hline & $\mathrm{N}=\mathbf{2 0}$ & & & & & \\
\hline $\begin{array}{l}\text { Alanine } \\
\text { transaminase }(\mathrm{IU} / \mathrm{L})\end{array}$ & $-0.7 \pm 2.96$ & $-1.05 \pm 3.93$ & $-1.19 \pm 3.17$ & $-1.1 \pm 3.23$ & 0.968 & 0.918 \\
\hline $\begin{array}{l}\text { Aspartate } \\
\text { transaminase (IU/L) }\end{array}$ & $0.05 \pm 2.48$ & $-0.8 \pm 2.48$ & $-0.09 \pm 2.39$ & $0 \pm 3.31$ & 0.708 & 0.426 \\
\hline Weight (kg) & $\begin{array}{l}-2.59 \pm \\
2.45\end{array}$ & $\begin{array}{l}-4.88 \pm \\
3.14^{\mathrm{b}}\end{array}$ & $\begin{array}{l}-4.88 \pm \\
2.63^{b}\end{array}$ & $\begin{array}{l}-5.79 \pm \\
2.77^{c}\end{array}$ & 0.004 & 0.004 \\
\hline $\begin{array}{l}\text { Body mass index } \\
\left(\mathrm{kg} / \mathrm{m}^{2}\right)\end{array}$ & $\begin{array}{l}-0.92 \pm \\
0.85\end{array}$ & $\begin{array}{l}-1.96 \pm \\
1.299^{b}\end{array}$ & $\begin{array}{l}-1.85 \pm \\
1.01^{\mathrm{b}}\end{array}$ & $\begin{array}{l}-2.09 \pm \\
1.17^{c}\end{array}$ & 0.004 & 0.006 \\
\hline $\begin{array}{l}\text { Waist circumstance } \\
\text { (cm) }\end{array}$ & $\begin{array}{l}-1.86 \pm \\
2.12\end{array}$ & $\begin{array}{l}-3.84 \pm \\
2.86\end{array}$ & $-2.95 \pm 3.35$ & $\begin{array}{l}-3.16 \\
\pm 2.55\end{array}$ & 0.154 & 0.26 \\
\hline $\begin{array}{l}\text { Hip circumstance } \\
\text { (cm) }\end{array}$ & $\begin{array}{l}-2.345 \pm \\
2.01\end{array}$ & $-4.83 \pm 4.16$ & $-4.08 \pm 4.08$ & $\begin{array}{l}-4.94 \\
\pm 3.37\end{array}$ & 0.081 & 0.161 \\
\hline $\begin{array}{l}\text { Physical Activity } \\
\left(\text { MET } \cdot \min \cdot \mathrm{wk}^{-1}\right)\end{array}$ & $\begin{array}{l}22.75 \\
(-74.25 \\
346)\end{array}$ & $\begin{array}{l}49.5 \\
(-738.75 \\
426)\end{array}$ & $\begin{array}{l}-213 \\
(-213,346.5)\end{array}$ & $\begin{array}{l}198 \\
(-178.5 \\
532)\end{array}$ & 0.763 & 0.98 \\
\hline \multicolumn{7}{|c|}{ MET $\cdot \min \cdot w^{-} 1$ : metabolic equivalent of task minute per week. } \\
\hline \multicolumn{7}{|c|}{$\begin{array}{l}\text { Data were presented as mean } \pm \text { standard deviation or median (interquartile (IQR)) for normally and } \\
\text { non-normally distributed data, respectively. }\end{array}$} \\
\hline \multicolumn{7}{|c|}{$\begin{array}{l}\text { P was calculated for the comparison variables between four groups using one-way analysis of } \\
\text { variance (one-way ANOVA) with post hoc (LSD) analysis. }\end{array}$} \\
\hline \multicolumn{7}{|c|}{ \# $\mathrm{P}$ was calculated for the comparison variables between four groups using Kruskal-Wallis. } \\
\hline \multicolumn{7}{|c|}{$\begin{array}{l}\text { *Adjusted P-value was calculated using ANCOVA or nonparametric ANCOVA; Adjusted for physical } \\
\text { activity levels at the baseline. }\end{array}$} \\
\hline
\end{tabular}

The comparison of lipid profiles within and between groups was depicted in Table 3. Moreover, the results of the pairwise comparisons of serum lipid profiles for ANOVA and ANCOVA were showed in supplementary file, Table S.3. 
Table 3

Comparisons of lipid profiles of the participants between and within the groups.

Variables

Groups

p

Adjusted

Placebo

Curcumin

Zinc

$(\mathrm{N}=20)$

$(\mathrm{N}=21)$

$(\mathrm{N}=21)$

Zinc \&

curcumin

$(\mathrm{N}=20)$

Triglyceride (mg/dl)

\begin{tabular}{lllllll}
$\begin{array}{l}\text { Pre- } \\
\text { intervention }\end{array}$ & $\begin{array}{l}132.55 \pm \\
25.96\end{array}$ & $\begin{array}{l}131.48 \pm \\
26.16\end{array}$ & $\begin{array}{l}125.76 \pm \\
25.74\end{array}$ & $126.1 \pm 27.64$ & 0.776 & - \\
\hline $\begin{array}{l}\text { Post- } \\
\text { intervention }\end{array}$ & $123.1 \pm 18.6$ & $\begin{array}{l}107.48 \pm \\
11.9\end{array}$ & $\begin{array}{l}114.52 \\
\pm 16.93\end{array}$ & $111.6 \pm 18.01$ & 0.026 & 0.286 \\
\hline P-value & $\mathbf{0 . 0 0 1}$ & $<\mathbf{0 . 0 0 1}$ & $\mathbf{0 . 0 0 1}$ & $<0.001$ & - & - \\
\hline Change & $-9.45 \pm$ & $-24.0 \pm$ & $-11.24 \pm$ & $-14.50 \pm$ & $\mathbf{0 . 0 0 7}$ & $\mathbf{0 . 0 0 1}$ \\
& 10.51 & $18.97^{\mathrm{b}}$ & 13.821 & 12.094 & &
\end{tabular}

Total. Cholesterol (mg/dl)

$\begin{array}{lllllll}\text { Pre- } & 186.5 \pm & 188.95 \pm & 182.52 \pm & 181.35 \pm & 0.716 & - \\ \text { intervention } & 24.91 & 23.5 & 26.11 & 19.98 & & \\ \text { Post- } & 184.25 \pm & 175.71 \pm & 172.67 \pm & 170.95 \pm & 0.146 & 0.583 \\ \text { intervention } & 24.4 & 17.01 & 21.34 & 13.45 & & \end{array}$

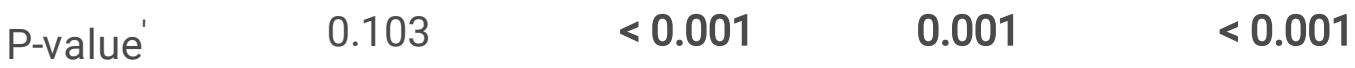

$\begin{array}{lllllll}\text { Change } & -2.25 \pm 5.87 & -13.24 & -9.86 \pm 10.94 & -10.40 \pm 9.98 & 0.005 & 0.15\end{array}$

$\operatorname{LDL}(\mathrm{mg} / \mathrm{dl})$

HDL: high-density lipoprotein-cholesterol; LDL: low-density lipoprotein; Non-HDL: non-high-density lipoprotein.

$P<0.05$ was considered significant difference.

Data were presented as mean \pm standard deviation.

'P-value was calculated for the comparison variables within group using paired t-test.

P-value was calculated for the comparison variables between four groups using one-way analysis of variance (one-way ANOVA) with post hoc (LSD) analysis.

*Adjusted P-value was calculated using ANCOVA; Adjusted for PA levels at the baseline, changes in $\mathrm{BMl}$ and weight.

Significant changes with placebo group indicated by ${ }^{a}$ adjusted $p<0.05,{ }^{b}$ adjusted $p \leq 0.01,{ }^{c}$ adjusted $\mathrm{p} \leq 0.001$. 


\begin{tabular}{|c|c|c|c|c|c|c|}
\hline \multirow[t]{2}{*}{ Variables } & \multicolumn{4}{|l|}{ Groups } & \multirow[t]{2}{*}{ p } & \multirow{2}{*}{$\begin{array}{l}\text { Adjusted } \\
\text { p * }\end{array}$} \\
\hline & $\begin{array}{l}\text { Placebo } \\
(\mathrm{N}=20)\end{array}$ & $\begin{array}{l}\text { Curcumin } \\
(\mathrm{N}=21)\end{array}$ & $\begin{array}{l}\text { Zinc } \\
(\mathrm{N}=21)\end{array}$ & $\begin{array}{l}\text { Zinc \& } \\
\text { curcumin } \\
(\mathrm{N}=20)\end{array}$ & & \\
\hline $\begin{array}{l}\text { Pre- } \\
\text { intervention }\end{array}$ & $\begin{array}{l}111.74 \pm \\
22.29\end{array}$ & $\begin{array}{l}114.63 \pm \\
20.22\end{array}$ & $\begin{array}{l}110.81 \pm \\
28.22\end{array}$ & $\begin{array}{l}110.87 \pm \\
20.53\end{array}$ & 0.944 & - \\
\hline $\begin{array}{l}\text { Post- } \\
\text { intervention }\end{array}$ & $\begin{array}{l}109.48 \pm \\
22.77\end{array}$ & $97.74 \pm 18.9$ & $\begin{array}{l}94.86 \pm \\
23.78\end{array}$ & $92.93 \pm 15.65$ & 0.057 & 0.378 \\
\hline P-value' & 0.155 & $<0.001$ & $<0.001$ & $<0.001$ & - & - \\
\hline Change & $-2.26 \pm 6.83$ & $\begin{array}{l}-16.89 \\
\pm 11.68^{b}\end{array}$ & $\begin{array}{l}-15.96 \pm \\
14.82^{\mathrm{a}}\end{array}$ & $\begin{array}{l}-17.93 \pm \\
10.06^{\mathrm{a}}\end{array}$ & $<.001$ & 0.035 \\
\hline \multicolumn{7}{|l|}{ HDL (mg/dl) } \\
\hline $\begin{array}{l}\text { Pre- } \\
\text { intervention }\end{array}$ & $48.25 \pm 5.58$ & $47 \pm 6.39$ & $47.14 \pm 6.77$ & $46 \pm 6.37$ & 0.734 & - \\
\hline $\begin{array}{l}\text { Post- } \\
\text { intervention }\end{array}$ & $50.15 \pm 5.34$ & $\begin{array}{l}56.47 \pm \\
6.14^{\mathrm{b}}\end{array}$ & $54.9 \pm 6.8^{\mathrm{a}}$ & $55.7 \pm 6.3^{\mathrm{a}}$ & 0.007 & 0.016 \\
\hline P-value' & $<0.001$ & $<0.001$ & $<0.001$ & $<0.001$ & - & - \\
\hline Change & $1.90 \pm 1.41$ & $9.48 \pm 4.14^{c}$ & $7.76 \pm 6.40^{c}$ & $9.70 \pm 3.23^{c}$ & $\begin{array}{l}<.001 \\
0.00\end{array}$ & $<0.001$ \\
\hline \multicolumn{7}{|c|}{ Non-HDL (mg/dl) } \\
\hline $\begin{array}{l}\text { Pre- } \\
\text { intervention }\end{array}$ & $\begin{array}{l}138.25 \pm \\
26.47\end{array}$ & $\begin{array}{l}141.95 \pm \\
24.91\end{array}$ & $\begin{array}{l}135.38 \pm \\
26.47\end{array}$ & $\begin{array}{l}135.35 \pm \\
21.81\end{array}$ & 0.834 & - \\
\hline
\end{tabular}

HDL: high-density lipoprotein-cholesterol; LDL: low-density lipoprotein; Non-HDL: non-high-density lipoprotein.

$P<0.05$ was considered significant difference.

Data were presented as mean \pm standard deviation.

'P-value was calculated for the comparison variables within group using paired t-test.

P-value was calculated for the comparison variables between four groups using one-way analysis of variance (one-way ANOVA) with post hoc (LSD) analysis.

*Adjusted P-value was calculated using ANCOVA; Adjusted for PA levels at the baseline, changes in $\mathrm{BMl}$ and weight.

Significant changes with placebo group indicated by ${ }^{a}$ adjusted $p<0.05,{ }^{b}$ adjusted $p \leq 0.01,{ }^{c}$ adjusted $\mathrm{p} \leq 0.001$. 


\begin{tabular}{|c|c|c|c|c|c|c|}
\hline \multirow[t]{2}{*}{ Variables } & \multicolumn{4}{|l|}{ Groups } & \multirow[t]{2}{*}{$\mathbf{p}$} & \multirow{2}{*}{$\begin{array}{l}\text { Adjusted } \\
\text { p * }\end{array}$} \\
\hline & $\begin{array}{l}\text { Placebo } \\
(\mathrm{N}=20)\end{array}$ & $\begin{array}{l}\text { Curcumin } \\
(\mathrm{N}=21)\end{array}$ & $\begin{array}{l}\text { Zinc } \\
(\mathrm{N}=21)\end{array}$ & $\begin{array}{l}\text { Zinc \& } \\
\text { curcumin } \\
(\mathrm{N}=20)\end{array}$ & & \\
\hline $\begin{array}{l}\text { Post- } \\
\text { intervention }\end{array}$ & $\begin{array}{l}134.1 \\
\pm 26.47\end{array}$ & $\begin{array}{l}119.24 \pm \\
24.91\end{array}$ & $\begin{array}{l}117.76 \pm \\
31.79\end{array}$ & $\begin{array}{l}115.25 \pm \\
21.81\end{array}$ & 0.036 & 0.34 \\
\hline P-value' & 0.009 & $<0.001$ & $<0.001$ & $<0.001$ & - & - \\
\hline Change & $-4.15 \pm 6.42$ & $\begin{array}{l}-22.71 \pm \\
13.27^{c}\end{array}$ & $\begin{array}{l}-17.62 \pm \\
15.14^{\mathrm{a}}\end{array}$ & $\begin{array}{l}-20.10 \pm \\
10.27^{\mathrm{a}}\end{array}$ & $\hat{0} .001$ & 0.003 \\
\hline \multicolumn{7}{|c|}{ HDL to LDL ratio } \\
\hline $\begin{array}{l}\text { Pre- } \\
\text { intervention }\end{array}$ & $26.51 \pm 5.19$ & $24.94 \pm 6.85$ & $24.57 \pm 6.11$ & $24.48 \pm 6.09$ & 0.712 & 0.192 \\
\hline $\begin{array}{l}\text { Post- } \\
\text { intervention }\end{array}$ & $24.62 \pm 3.72$ & $21.49 \pm 2.38$ & $22.9 \pm 3.39$ & $22.32 \pm 3.6$ & 0.025 & 0.132 \\
\hline P-value' & 0.01 & $<0.001$ & $<0.001$ & $<0.001$ & - & - \\
\hline Change & $-0.03 \pm 0.04$ & $-0.18 \pm 0.1^{c}$ & $-0.16 \pm 0.15^{b}$ & $-0.19 \pm 0.08^{c}$ & $\hat{0} .001$ & 0.002 \\
\hline \multicolumn{7}{|c|}{$\begin{array}{l}\text { HDL: high-density lipoprotein-cholesterol; LDL: low-density lipoprotein; Non-HDL: non-high-density } \\
\text { lipoprotein. }\end{array}$} \\
\hline \multicolumn{7}{|c|}{$P<0.05$ was considered significant difference. } \\
\hline \multicolumn{7}{|c|}{ Data were presented as mean \pm standard deviation. } \\
\hline \multicolumn{7}{|c|}{ 'P-value was calculated for the comparison variables within group using paired t-test. } \\
\hline \multicolumn{7}{|c|}{$\begin{array}{l}\text { P-value was calculated for the comparison variables between four groups using one-way analysis of } \\
\text { variance (one-way ANOVA) with post hoc (LSD) analysis. }\end{array}$} \\
\hline \multicolumn{7}{|c|}{$\begin{array}{l}\text { *Adjusted P-value was calculated using ANCOVA; Adjusted for PA levels at the baseline, changes in } \\
\text { BMl and weight. }\end{array}$} \\
\hline $\begin{array}{l}\text { Significant } \mathrm{cl} \\
\text { adjusted } \mathrm{p} \leq\end{array}$ & $\begin{array}{l}\text { es with plac } \\
11 .\end{array}$ & group indic & by ${ }^{a}$ adjuste & $0.05,{ }^{b}$ adj & $0 \leq$ & \\
\hline
\end{tabular}

As set out in Table 3 (within-group comparisons), curcumin, zinc, and "curcumin \& zinc" groups revealed a significant improvement for post-intervention serum lipid profiles.

The placebo group showed a notable difference in post intervention TG $(p=0.001), H D L, H D L / L D L$ ratio $(p \leq 0.001)$, and non-HDL $(p=0.009)$ without any significant difference in TC $(p=0.155)$ and LDL $(p=$ 0.103) levels (Table 3). 
There was no significant difference in pre-intervention lipid profiles between the four groups.

After removing cofounders' effects (baseline PA and changes in BMI and weight), a remarkable difference was found only for post intervention HDL (adjusted $p=0.016$ ) between groups, without any significant difference for post-intervention TG (adjusted $p=0.286$ ), non-HDL (adjusted $p=0.34$ ), LDL (adjusted $p=$ 0.378 ), $T C$ (adjusted $p=0.583$ ), and HDL/ LDL ratio (adjusted $p=0.132$ ) between groups (Table 3 ).

Serum HDL levels significantly increased in the curcumin (adjusted $p=0.002)$, zinc (adjusted $p=0.016$ ), and "curcumin \& zinc" (adjusted $p=0.015$ ) groups compared to the placebo group after adjusting cofounders' effects (supplementary file, Table S.3).

After removing the confounders' effect (baseline PA and changes in BMI and weight), the changes of all lipid profiles (pre-post) including serum TG (adjusted $p=0.001$ ), LDL (adjusted $p=0.035$ ), HDL (adjusted $p \leq 0.001$ ), non-HDL (adjusted $p=0.003$ ), and HDL/LDL ratio (adjusted $p=0.002$ ) except serum TC (adjusted $p=0.15$ ) showed a significant difference between four groups. In compared to the placebo group, three supplement groups sustained a remarkable improvement for $L D L, H D L$, non-HD, and HDL/LDL ratio after removing the cofounders' effects. The p-values were depicted in Table S.3 (supplementary file). After removing the cofounders' effects, changes (pre-post) in TG were revealed a significant difference only in the curcumin group in comparison to the placebo group (adjusted $p=$ 0.001).

\section{Discussion}

The main results of the present 90-days trial indicated that there was a significant difference between the groups for 1) the changes in BMI, weight, TG, LDL, HDL, non-HDL, and HDL/LDL ratio and 2) postintervention HDL. Given that no significant difference for changes in ALT and AST and any reports of severe adverse reactions during the trial, the safety of the prescribed combination was represented.

Despite lifestyle recommendations, hypocaloric diet, and ongoing patient follow-up, the mean decrease in energy in each group was less than 500-1000 kcal (the values of recommended) and the changes in PA and dietary intake were no significant between groups, but the change (pre-post intervention) in weight and BMI showed a shift towards their improvement. Therefore, it may emanate from the positive effects of supplements on weight and BMI. It is notable that the undesirable impacts of quarantine on lowering PA cannot be ignored due to the prohibition of leaving home and the closure of gym, fitness and sports club, and park and the restrictions on social activity 26,27 .

It seems under the supervision of a nutritionist along with the closure of bars, restaurants, and coffee shops, remote staff, and enough time to prepare foods in the home due to COVID-19 quarantine may have led to the healthy selection of foods (better quality) without the remarkable changes in the amount of food (quantify) which contributed to the improvement of some of serum lipid profiles in the placebo groups. 
After adjusting cofounder effects, the post-intervention comparisons of the serum lipid profiles showed there was a significant difference only for serum HDL between groups. This finding can presented the important role of cofounders as well as the supplements on serum lipid levels during the intervention.

The progression of pre-diabetes to T2DM emanates from oncoming disturbed glucose and lipid metabolism ${ }^{5}$. On the other hand, the previous studies illustrated a high prevalence of dyslipidemia in prediabetes and $\mathrm{T} 2 \mathrm{DM}^{3,4}$. However, a correlation was reported between dyslipidemia prevalence with age, sex, education level, smoking status, alcohol drinking status, and obesity ${ }^{4}$, the mean of the aforementioned variables was similar among all participants in the present trial. Therefore, the present findings may be related to the effect of the supplements.

As previously published ${ }^{11}$, curcumin and zinc co-supplementation significantly improved some glycemic parameters such as IR and IS. Therefore, in addition to their indirect effects on lipid profiles via the improvement of IR and IS, other mechanisms may be illustrated the positive effects of the supplements on the lipid profile.

Given that the control cofounder in the present trial, it can be stated that the studied supplementations were presented the main role in improving the serum lipid profiles.

In agreement with the present results, a meta-analysis (2017) showed curcumin and turmeric decrease the risk factors of CVD through lowering TG and LDL in pre-diabetes, metabolic syndrome, T2DM, and hypertension ${ }^{10}$. Moreover, a trial conducted by Panahi et al. ${ }^{8}$, touched upon an improvement in the changes of serum HDL, TC, and non-HDL and but no significant difference in TG and LDL changes after 1000 mg-curcumin intake in patients with T2DM. In another trial carried out by Thota et al. ${ }^{28}$, the curcumin alone $(1000 \mathrm{mg})$ or curcumin $(1000 \mathrm{mg})$ with omega-3 did not alter serum lipid profiles in the patients with IFG and IGT.

In some previous investigations, an improvement in some serum lipid levels (TC, HDL, LDL, and TG) was illustrated following zinc supplementation $15,17,18$ in different populations. Furthermore, the findings of a meta-analysis conducted by Asbaghi et al. ${ }^{17}$ presented the beneficial impacts of zinc supplementation on HDL in both studies duration less or more than 12-weeks. Also, zinc supplementation decreased serum $T G, T C$, and LDL only in the studies with 12 weeks or less. It is notable that they pointed out the highest impact of zinc supplementation was on a dosage of less than $100 \mathrm{mg}{ }^{17}$. While the present trial did not show the beneficial effects of zinc supplement alone on serum TC and LDL and changes of TG in comparison to the placebo group, zinc supplementation with curcumin improved all serum lipid profiles except serum TC levels compared to the placebo group.

The conflicting findings of zinc or curcumin effects on serum lipid profiles can emanate from bioavailability and dose of the supplement, study duration, variety in the studied population (race, age, sex, history of diseases and etc.), several methodological limitations, the usage of supplement alone or with other nutrients (co-supplementation). 
Some mechanisms that can justify the effect of curcumin or zinc supplements are separately described: Curcumin can reduce serum TG through the different mechanisms, including increasing the gene expression of adiponectin and peroxisome proliferator-activated receptor alpha (PPAR-a), peroxisome proliferator-activated receptor gamma (PPAR- $\gamma$ ), cholesteryl ester transfers protein, and lipoprotein lipase activity. Curcumin can also reduce serum LDL by suppressing the LDL receptor gene expression through enhancement in PPAR-y activation. In addition, curcumin reduces TC by affecting enzyme pathways of cholesterol metabolism ${ }^{29}$.

Zinc may play effective roles in serum lipid profiles through several molecular mechanisms. The proposed mechanisms of directly and indirectly zinc action are as follows: 1) zinc can affect the activity of pancreatic $\beta$-cells through transporter expression; hence, regulate insulin storage and secretion; 2 ) zinc can improve insulin sensitivity or insulin resistance through a) increasing the phosphorylation of insulinreceptor substrates at the adipocytes ${ }^{12}, \mathrm{~b}$ ) lipolysis inhibition in adipose tissues, which lead to a decrease in fatty acids released and finally regulate lipoprotein synthesis (VLDL and LDL secretion) from the liver; and 3) zinc can affect the gene expression of enzymes involved in hepatic lipid homeostasis leading to regulate lipid synthesis and utilization in mitochondria and peroxisomes ${ }^{30}$.

The main limitation of the present trial was the participant recruitment was from single-center and the single dosage of each supplement was used. Further, a part of the research was done during quarantine condition (COVID-19), the quarantine may have a desirable and undesirable impact on the present trial. The present study is unique in its novelty due to the use of the zinc and curcumin co-supplements on serum lipid profiles of pre-diabetic patients.

It was suggested to conduct further RCTs with regard to the recruitment of patients with a longer history of pre-diabetes due to the appearance of macro and microvascular complications of pre-diabetes may need to longer time (the duration mean (Cl) of pre-diabetes diagnosis in this trial was $2.32(2.02,2.62)$ months). Moreover, the serum lipid profiles of participants at the baseline were in the normal range. The different results may find in recruiting the participants with an abnormality in serum lipid profiles at the baseline.

\section{Conclusion}

The present trial was reported the beneficial effects of curcumin and zinc co-supplementation on serum HDL and the changes in serum TG, LDL, HDL, non-HDL, HDL/LDL ratio, BMI, and weight among patients with diabetes. Therefore, it may play a potential role in the prevention of macro and microvascular complications.

It is suggested that multicenter and long-term clinical trials be conducted to confirm the serum lipidimproving properties of curcumin and zinc co-supplements in pre-diabetic patients with dyslipidemia. Moreover, it recommended examining whether co-administration of curcumin and zinc in other doses and forms can be useful for the prevention of diabetic macro and microvascular complications. 


\section{Abbreviations}

ADA, American Diabetes Association

ALT, Alanine Transaminase, ANCOVA, Analysis of Covariance

AST, Aspartate Aminotransferase

Body Mass Index

CONSORT, Consolidated Standards for Reporting Trials

DBP, Diastolic Blood Pressure

ELISA

Enzyme-Linked Immunosorbent Assay

FPG, Fasting Plasma Glucose

IPAQ-SF, Short form of International Physical Activity Questionnaire

$I Q R$, Interquartile range

IRCT, Iranian Registry of Clinical Trials

$\mathrm{IR}$, insulin resistance

IS, Insulin Sensitivity

LDL-C, Low-density lipoprotein cholesterol

MET, Metabolic Equivalent of Task

Non-HDL, non-high-density lipoprotein-cholesterol

One-way ANOVA, One-way Analysis of variance

PA, physical activity

PPAR-a, Peroxisome proliferator-activated receptor alpha

PPAR- $\gamma$, Peroxisome proliferator-activated receptor gamma

RCT, Randomized Clinical Trial

SBP, Systolic Blood Pressure

SD, Standard Deviation

SPSS, Statistical Software Package

T2DM, Type 2 Diabetes Mellitus.

\section{Declarations}

\section{Ethics approval and consent to participate}

This trial was approved by the Medical Ethics Committee of Ahvaz Jundishapur University of Medical Sciences, Ahvaz, Iran (Ethical code: IR.AJUMS.REC.1398.504) in accordance with the ethical standards of the institutional and/or national research committee and with the 1964 Helsinki declaration and its later amendments or comparable ethical standards. The written informed consent was obtained from all patients to participate in this study after receiving a complete explanation of the study protocols and objectives.

\section{Consent for publication}


The authors affirm that human research participants provided informed consent for publication of their data.

\section{Availability of data and materials}

The datasets used and/or analyzed during the current study are available from the corresponding author on reasonable request.

\section{Competing interests}

The authors declare no competing interests associated with this publication and there has been no significant financial support for this work that could have influenced its outcome.

\section{Funding}

The project was financially supported by Ahvaz Jundishapur University of Medical Sciences under the research grant number 8913 dated October 20, 2019. The grant covered patient recruitment, manpower, consumables, and other runs in costs for the trial. The funding body did not have any involvement in the study design, the execution, data collection, data analysis, and interpretation, or preparation and publishing of the present manuscript.

\section{Authors' Contribution}

Majid Karandish, Hassan Mozaffari-khosravi, and Maryam Azhdari participated in study design and protocol development. Maryam Azhdari and Seyed Mohammad Mohammadi coordinated recruitment and participant management. Seyed Mohammad Mohammadi confirmed all participants. Maryam Azhdari collected data adhering to study protocol. Bahman Cheraghian provided statistical advice and input. The initial draft was written by Maryam Azhdari. All authors read and approved the final manuscript.

\section{Acknowledgements}

The authors sincerely thank all patients participating in this study. Theauthors also extend our appreciation to Dr.Khalilzadeh who was the chief of the Diabetes Research and Clinical Practice Center of Yazd, Iran due to his cooperation in every way possible in this study. The authors are grateful to " $\mathrm{M} / \mathrm{s}$ Arjuna Natural Pvt Ltd., India and "Dineh company, Iran". Moreover, the present manuscript is extracted from Ph.D. thesis of Maryam Azhdari.

\section{References}

1. 2. Classification and Diagnosis of Diabetes: Standards of Medical Care in Diabetes-2020. Diabetes care. Jan 2020;43(Suppl 1):S14-s31. doi:10.2337/dc20-S002. 
2. Mahat RK, Singh N, Arora M, Rathore V. Health risks and interventions in prediabetes: A review. Diabetes \& metabolic syndrome. Jul-Aug. 2019;13(4):2803-11. doi:10.1016/j.dsx.2019.07.041.

3. Al Amri T, Bahijri S, Al-Raddadi R, et al. The Association Between Prediabetes and Dyslipidemia Among Attendants of Primary Care Health Centers in Jeddah, Saudi Arabia. Diabetes metabolic syndrome obesity: targets therapy. 2019;12:2735-43. doi:10.2147/dmso.s233717.

4. Li Y, Zhao L, Yu D, Ding G. The prevalence and risk factors of dyslipidemia in different diabetic progression stages among middle-aged and elderly populations in China. PLoS One. 2018;13(10):e0205709.

5. Erion DM, Park HJ, Lee HY. The role of lipids in the pathogenesis and treatment of type 2 diabetes and associated co-morbidities. BMB reports Mar. 2016;49(3):139-48. doi:10.5483/bmbrep.2016.49.3.268.

6. Azhdari M, Karandish M, Mansoori A. Metabolic benefits of curcumin supplementation in patients with metabolic syndrome: A systematic review and meta-analysis of randomized controlled trials. Phytother Res. 2019;33(5):1289-301.

7. Omosa L, Midiwo J, Kuete V. Curcuma longa. In: Medicinal Spices and Vegetables from Africa. Elsevier; 2017. pp. 425-35.

8. Panahi Y, Khalili N, Sahebi E, et al. Curcuminoids modify lipid profile in type 2 diabetes mellitus: a randomized controlled trial. Complement Ther Med. 2017;33:1-5.

9. Azhdari M, Zilaee M, Karandish M, et al. Red vine leaf extract (AS 195) can improve some signs and symptoms of chronic venous insufficiency, a systematic review. Phytotherapy Research. 2020.

10. Qin S, Huang L, Gong J, et al. Efficacy and safety of turmeric and curcumin in lowering blood lipid levels in patients with cardiovascular risk factors: a meta-analysis of randomized controlled trials. Nutrition journal. 2017;16(1):68.

11. Karandish M, Mozaffari-khosravi H, Mohammadi SM, Cheraghian B, Azhdari M. The effect of curcumin and zinc co-supplementation on glycemic parameters in overweight or obese prediabetic subjects: A phase 2 randomized, placebo-controlled trial with a multi-arm, parallel-group design. Phytotherapy Research. 2021.

12. Lynch CJ, Patson BJ, Goodman SA, Trapolsi D, Kimball SR. Zinc stimulates the activity of the insulinand nutrient-regulated protein kinase mTOR. American Journal of Physiology-Endocrinology Metabolism. 2001;281(1):E25-34.

13. Dunn MF. Zinc-ligand interactions modulate assembly and stability of the insulin hexamer-a review. Biometals. 2005;18(4):295-303.

14. Stefanidou M, Maravelias C, Dona A, Spiliopoulou C. Zinc: a multipurpose trace element. Archives of Toxicology. 2005/09/27 2005;80(1):1. doi:10.1007/s00204-005-0009-5.

15. Jayawardena R, Ranasinghe P, Galappatthy P, Malkanthi R, Constantine G, Katulanda P. Effects of zinc supplementation on diabetes mellitus: a systematic review and meta-analysis. Diabetol Metab Syndr. 2012;4(1):13. 
16. Choi S, Liu X, Pan Z. Zinc deficiency and cellular oxidative stress: prognostic implications in cardiovascular diseases. Acta Pharmacologica Sinica. 2018

/07/01

2018;39(7):1120-1132. doi:10.1038/aps.2018.25.

17. Asbaghi O, Sadeghian M, Fouladvand F, et al. Effects of zinc supplementation on lipid profile in patients with type 2 diabetes mellitus: A systematic review and meta-analysis of randomized controlled trials. Nutrition, Metabolism and Cardiovascular Diseases. 2020.

18. Ranasinghe $\mathrm{P}$, Wathurapatha WS, Ishara $\mathrm{MH}$, et al. Effects of Zinc supplementation on serum lipids: a systematic review and meta-analysis. Nutrition metabolism. 2015;12:26. doi:10.1186/s12986-0150023-4.

19. Jafarnejad S, Mahboobi S, McFarland LV, Taghizadeh M, Rahimi F. Meta-Analysis: Effects of Zinc Supplementation Alone or with Multi-Nutrients, on Glucose Control and Lipid Levels in Patients with Type 2 Diabetes. Preventive nutrition and food science. Mar 2019;24(1):8-23. doi:10.3746/pnf.2019.24.1.8.

20. Karandish M, Mozaffari-Khosravi H, Mohammadi SM, Azhdari M, Cheraghian B. Evaluation of the effect of curcumin and zinc co-supplementation on glycemic measurements, lipid profiles, and inflammatory and antioxidant biomarkers in overweight or obese prediabetic patients: a study protocol for a randomized double-blind placebo-controlled phase 2 clinical trial. Trials. 2020;21(1):111.

21. Panahi Y, Khalili N, Sahebi E, et al. Effects of curcuminoids plus piperine on glycemic, hepatic and inflammatory biomarkers in patients with type 2 diabetes mellitus: a randomized double-blind placebo-controlled trial. Drug research. 2018;68(07):403-9.

22. Kim J, Ahn J. Effect of zinc supplementation on inflammatory markers and adipokines in young obese women. Biol Trace Elem Res. 2014;157(2):101-6.

23. Craig CL, Marshall AL, Sjöström M, et al. International physical activity questionnaire: 12-country reliability and validity. Medicine science in sports exercise. 2003;35(8):1381-95.

24. Pickering $T$, Hall J, Appel $L$, et al. Recommendations for blood pressure measurement in humans. A statement for professionals from the subcommittee of Professional and Public Education of the American Heart Association Council on High Blood Pressure Research. Hypertension. 2005;45:14261.

25. Rifai N, Bachorik PS, Albers JJ. Lipids, lipoproteins and apolipoproteins. Tietz textbook of clinical chemistry. 1999;3:809-61.

26. Kriaucioniene V, Bagdonaviciene L, Rodríguez-Pérez C, Petkeviciene J. Associations between changes in health behaviours and body weight during the COVID-19 quarantine in Lithuania: the Lithuanian COVIDiet Study. Nutrients. 2020;12(10):3119.

27. López-Sánchez GF, López-Bueno R, Gil-Salmerón A, et al. Comparison of physical activity levels in Spanish adults with chronic conditions before and during COVID-19 quarantine. Eur J Pub Health. 2020. doi:10.1093/eurpub/ckaa159. 
28. Thota RN, Acharya SH, Garg ML. Curcumin and/or omega-3 polyunsaturated fatty acids supplementation reduces insulin resistance and blood lipids in individuals with high risk of type 2 diabetes: a randomised controlled trial. Lipids Health Dis. 2019;18(1):31.

29. Panahi Y, Ahmadi Y, Teymouri M, Johnston TP, Sahebkar A. Curcumin as a potential candidate for treating hyperlipidemia: a review of cellular and metabolic mechanisms. Journal of cellular physiology. 2018;233(1):141-52.

30. Dieck Ht, Döring F, Fuchs D, Roth H-P, Daniel H. Transcriptome and proteome analysis identifies the pathways that increase hepatic lipid accumulation in zinc-deficient rats. J Nutr. 2005;135(2):199205.

\section{Figures}




\section{O N S O R T}

TRANSPARENT REPORTING of TRIALS

\section{CONSORT 2010 Flow Diagram}

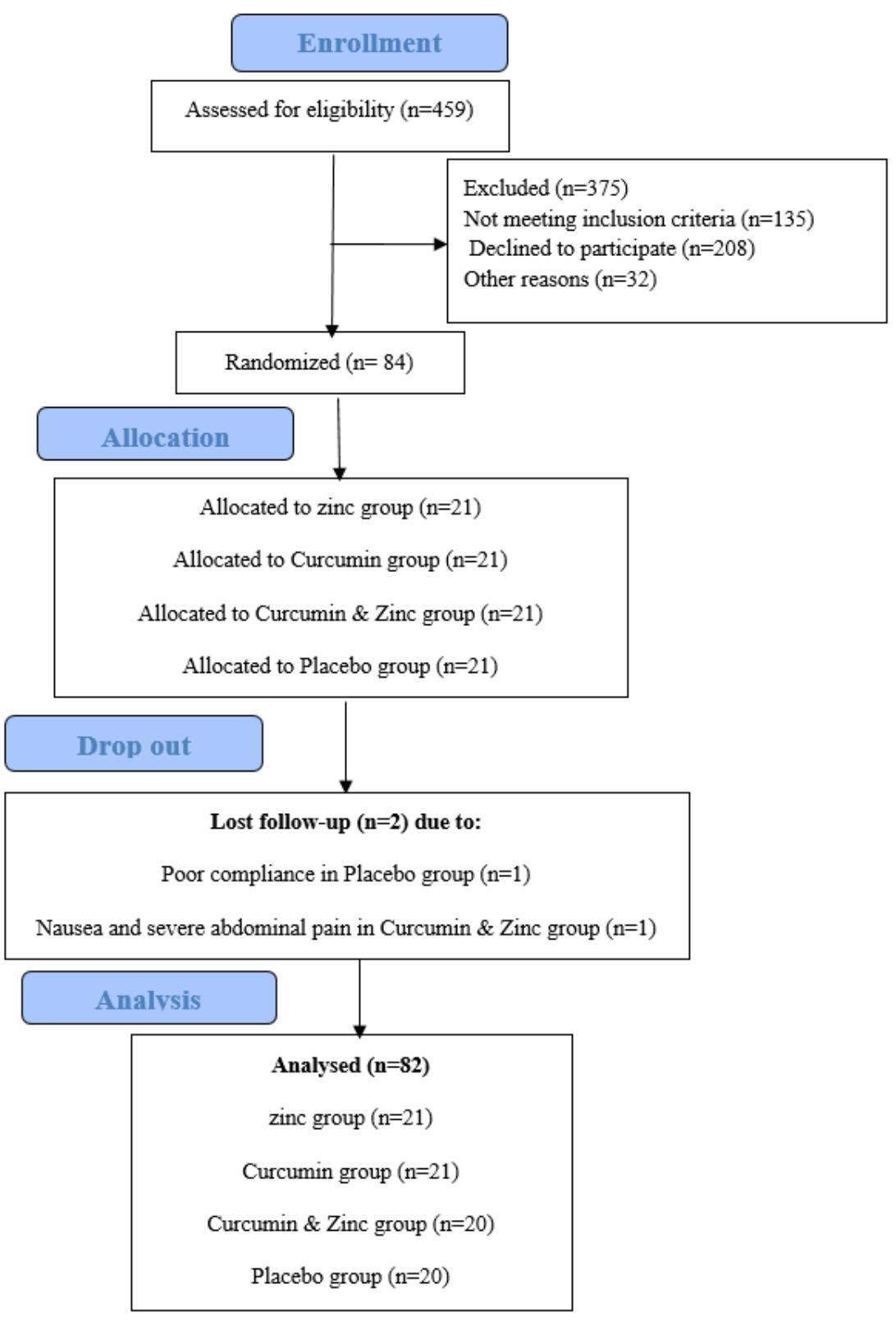

Figure 1

Consolidated Standards of Reporting Trials (CONSORT) flow chart- trial protocol

\section{Supplementary Files}

This is a list of supplementary files associated with this preprint. Click to download. 
- Supplementaryfiles.doc

Page 22/22 